\title{
Rheology of Copolymer Solutions. III. The Linear Viscoelasticity of SBS Block Copolymer Solutions
}

\author{
Kunihiro Osaki, Bong-Shik Kim, ${ }^{*}$ and Michio Kurata** \\ Institute for Chemical Research, Kyoto University Uji, Kyoto 611, Japan.
}

(Received December 21, 1977)

\begin{abstract}
Linear viscoelastic behavior was investigated for concentrated solutions of a styrene-butadiene block copolymer, that is, a 4-armed star-branched polybutadiene structure with a polystyrene block on each chain end. The effect of varying solvent, concentration $c$, and temperature $T$ was investigated by applying the method of reduced variables to the shear stresses at the start and on cessation of steady shear flow. When the solvent had a strong solvent power for both the polystyrene and the polybutadiene, the viscoelastic behavior was only slightly different from that in homopolymer solutions. In the case of the 1-chlorohexadecane solvent, the solubility of polystyrene varied much in the temperature range of measurement. In this case, the method of reduced variables with respect to $t-c$ and $t-T$ was not applicable, the viscosity $\eta^{0}$ varied to a very large extent with $c$ and $T$, and the ratio $\tau_{1}{ }^{0} / \eta^{0}$ increased with increasing $c$, where $\tau_{1}{ }^{0}$ is the maximum relaxation time. The result was tentatively interpreted in terms of a molecular aggregate consisting of a few molecules connected to each other through the hard domains of the polystyrene block solution.

KEY WORDS Block Copolymer / SBS / Solution / Linear Viscoeelasticity / Relaxation Time / Viscosity / Microdomain /
\end{abstract}

Block copolymers exhibit interesting mechanical behavior in bulk as well as in solutions. The characteristic features of mechanical behavior in the solid state should be understood in relation to the microdomain structures induced by the micro-phase separation of the block components. There are some reasons to believe that similar structures exist also in melts and solutions, ${ }^{1,2}$ although it is much more difficult to obtain definite proof of the existence of structures than is in the case of the solid state. Some of the rheological properties of solutions and melts are also believed to be understood in terms of microdomain structures. ${ }^{3-11}$ So far rheological studies on melts and solutions of block copolymers have been restricted mostly to measurements of the steady shear viscosity and the dynamic mechanical behavior at small

\footnotetext{
* Present Address: Department of Industrial Chemistry, College of Engineering, Yeungnam University, Gyongsan, Dae-Dong, Korea.

** To whom reprints should be requested.
}

deformation. Normal stress measurements by Kotaka and White seem to have led to a deeper insight into the microdomain structure and flow properties of solutions of block copolymers. ${ }^{8}$

On the other hand, measurements of rheological properties in various nonsteady flow histories have been performed on melts and solutions of homopolymers ${ }^{12}$ and on disperse systems of solid particles in polymer solutions. ${ }^{13}$ Constitutive models have been proposed and examined on the measured results in order to describe the mechanical properties with a unified equation. ${ }^{14}$ In the series of studies, we apply the methods of rheological studies developed for homopolymers and dispersed systems and see how they are effective for solutions of block copolymers. Preliminary reports have been given elsewhere. ${ }^{15,16}$ In this paper, we report on the effect of varying solvent, temperature, and concentration on the linear viscoelastic functions, zero-shear viscosity, and relaxation time of solutions of a styrenebutadiene block copolymer. 


\section{MATERIALS}

Commercial butadiene-styrene block copolymers from the Phillips Petroleum Company were used in the series of studies. We have reported here on a part of the results obtained for solutions of Solprene 411 in various solvents. Solprene 411 is a 4-armed star-branched block copolymer with a structure of a styrene block on the end of each branch of a 4-armed star-branched polybutadiene. A slight amount of a linear styrenebutadiene block copolymer corresponding to one branch may be included. ${ }^{17}$ In spite of the complicated structure of the molecule, we will sometimes denote the sample as SBS to emphasize that all the polystyrene blocks are located at the end of each chain. The number-average molecular weight of the polymer was evaluated as 185,000 from the osmotic pressure measurement. ${ }^{18}$ The content of styrene was $31.0 \mathrm{wt} \%{ }^{18}$ The molecular weight of the polystyrere block was calculated as 14,350 from the molecular weight, composition, and the supposed structure. This value was in fair agreement with the value 13,000 obtained with the gel permeation chromatograpy method after decomposing the polybutadiene chain by tert-butyl hydroperoxide.

Solutions in four solvents were studied: Liquid paraffin (Wako Chemical Industries, Ltd.; abbreviated as LP), 1-chlorohexadecane (Tokyo Chemical Industry Co., Ltd.; cetyl chloride, CC), di(2-ethylhexyl) phthalate (Nakarai Chemicals, Ltd.; dioctyl phthalate, DOP), and chlorinated biphenyl (Kanechlor 500 from Kanegafuchi Chemical Industry Co., Ltd.; CB). Polybutadiene was soluble in all these solvents. LP was a nonsolvent for polystyrene. CC was a solvent for polystyrene at high temperatures; a polystyrene of molecular weight 19,800 (standard polystyrene, batch 2a from Pressure Chemical Co.) dissolved in this solvent above $30^{\circ} \mathrm{C}$ and precipitated from the solution below $25^{\circ} \mathrm{C}$; a polystyrene of molecular weight 51,000 (standard polystyrene, batch 7a) dissolved above $50^{\circ} \mathrm{C}$ and precipitated below. The SBS sample could be dissolved in CC transparently at any concentration; the solution was colorless in the temperature range of $10-40^{\circ} \mathrm{C}$. DOP was probably a theta solvent for polystyrene; polystyrene samples of molecular weights 19,800 and 51,000 readily dissolved in this solvent in the tested range of $0-50^{\circ} \mathrm{C}$ and the solution of polystyrene of higher molecular weight $\left(\mathrm{ca} .10^{6}\right)$ became turbid below $10^{\circ} \mathrm{C}$. CB was a good solvent for polystyrene at all temperatures tested.

Polymer solutions were prepared in the following manner. Weighed amounts of polymer and solvent were dissolved in dichloromethane. Then the latter was evaporated mostly at room temperature. A slight amount (few per cent) of dichloromethane was evaporated at $50^{\circ} \mathrm{C}$ in vacuo. Dichloromethane was eliminated in about an hour and the loss of solvent was negligibly small.

\section{MEASUREMENTS AND METHOD OF STUDY}

We measured the shear stresses at the start and on cessation of shear flow of constant rate of shear. Suppose a shear flow of the rate of shear $\dot{\gamma}$ is started at time 0 . We denote the shear stress for $t \geq 0$ as $\sigma_{\mathrm{g}}(t, \dot{\gamma})$ and define a viscosity growth function by

$$
\bar{\eta}(t, \dot{\gamma})=\frac{\sigma_{\mathrm{g}}(t, \dot{\gamma})}{\dot{\gamma}}
$$

One can define also a viscosity decay function by

$$
\widetilde{\eta}(t, \dot{\gamma})=\frac{\sigma_{\mathrm{d}}(t, \dot{\gamma})}{\dot{\gamma}}
$$

where $\sigma_{\mathrm{d}}(t, \dot{\gamma})$ is the shear stress at time $t$ following a sudden cessation of steady shear flow. The steady shear viscosity $\eta(\dot{\gamma})$ is related to these functions by

$$
\eta(\dot{\gamma})=\lim _{t \rightarrow \infty} \bar{\eta}(t, \dot{\gamma})=\lim _{t \rightarrow 0} \tilde{\eta}(t, \dot{\gamma})
$$

At the limit of small $\dot{\gamma}$, the functions $\bar{\gamma}(t, \dot{\gamma})$, $\tilde{\eta}(t, \dot{\gamma})$, and $\eta(\dot{\gamma})$ become independent of $\dot{\gamma}$. We denote the limiting quantities by $\bar{\eta}(t), \tilde{\eta}(t)$, and $\eta^{0}$. The phenomenological theory of linear viscoelasticity is applicable to these quantities.

In spite of the fact that the linear viscoelastic functions $\bar{\eta}(t)$ and $\tilde{\eta}(t)$ are easily measured with conventional rheometers and are convenient quantities for studying polymeric liquids, they have not been studied very extensively. Some of the basic phenomenological relations concerning $\bar{\eta}(t)$ and $\tilde{\eta}(t)$ are as follows: ${ }^{19}$

$$
\bar{\eta}(t)+\tilde{\eta}(t)=\eta^{0}
$$


Rheology of Copolymer Solutions. III.

$$
\begin{gathered}
G(t)=\frac{\mathrm{d} \bar{\eta}}{\mathrm{d} t} \\
\tilde{\eta}(t)=\int_{-\infty}^{\infty} \tau H(\tau) \mathrm{e}^{-t / \tau} \mathrm{d} \ln \tau
\end{gathered}
$$

Here $\eta^{0}$ is the zero-shear viscosity, $G(t)$ is the relaxation modulus, and $H(\tau)$ is the distribution function of relaxation time $\tau$, or the relaxation spectrum. The relaxation spectrum and dynamic complex moduli can be calculated from the experimental results on $\bar{\eta}(t)$ or $\tilde{\eta}(t)$ with the use of approximation formulae. ${ }^{20}$ The steady shear compliance $J_{\mathrm{e}}{ }^{0}$ is given by

$$
J_{\mathrm{e}}^{0}=\frac{\int_{0}^{\infty} \tilde{\eta}(t) \mathrm{d} t}{\left(\eta^{0}\right)^{2}}
$$

It may be noted that a large part of the discussion presented by Kotaka and White ${ }^{8}$ is based on three quantities $\eta^{0}, J_{\mathrm{e}}^{0}$, and $\lambda \equiv \eta^{0} J_{\mathrm{e}}^{0}$. Thus by measuring the linear viscoelastic functions, we should be able to obtain results comparable to those of Kotaka and White, who measured the shear and normal stress components in steady shear flow. ${ }^{*}$

For later use, we express the linear viscoelastic functions in terms of the discrete distribution of relaxation times:

$$
\begin{gathered}
\tilde{\eta}(t)=\sum_{p} G_{\mathrm{p}}{ }^{0} \tau_{\mathrm{p}}{ }^{0} \mathrm{e}^{-t / \tau_{\mathrm{p}}{ }^{0}} \\
\bar{\eta}(t)=\sum_{p} G_{\mathrm{p}}{ }^{0} \tau_{\mathrm{p}}{ }^{0}\left(1-\mathrm{e}^{-t / \tau_{\mathrm{p}}{ }^{0}}\right) \quad\left(\tau_{1}{ }^{0}>\tau_{2}{ }^{0}>\tau_{3}{ }^{0} \cdots\right)
\end{gathered}
$$

where $\tau_{\mathrm{p}}{ }^{0}$ is the relaxation time and $G_{\mathrm{p}}{ }^{0}$ is the relaxation strength of the $p$-th relaxation mode. In examining the effect of varying concentration $c$ and temperature $T$, we employ the method of reduced variables. ${ }^{21}$ The method of reduced variables is applicable provided that the ratios

$$
\tau_{1}{ }^{0}: \tau_{2}{ }^{0}: \tau_{3}{ }^{0}: \cdots \quad \text { and } \quad G_{1}{ }^{0}: G_{2}{ }^{0}: G_{3}{ }^{0}: \cdots
$$

are independent of $c$ or $T$. Then the reduced functions

$$
\bar{\eta}_{\mathrm{r}}(t)=\bar{\eta}(t) \frac{\eta_{0}{ }^{0}}{\eta^{0}} \quad \text { and } \quad \tilde{\eta}_{\mathrm{r}}(t)=\tilde{\eta}(t) \frac{\eta_{0}{ }^{0}}{\eta^{0}}
$$

* The superscript 0 refers to a material constant related to the linear viscoelasticity throughout this paper. obtained for any value of $c$ or $T$ should be universal functions of a reduced time

$$
t_{\mathrm{r}}=t \frac{\tau_{10}^{0}}{\tau_{1}^{0}}
$$

and should agree with the functions obtained at the reference state, i.e., a certain (arbitrary) set of concentration $c_{0}$ and temperature $T_{0}$. The subscript 0 on $\eta^{0}$ and $\tau_{1}{ }^{0}$ implies the values for the reference state. Since the maximum relaxation time $\tau_{1}{ }^{0}$ cannot always be evaluated from experimental data, the factor $\tau_{10}^{0} / \tau_{1}{ }^{0}$ in eq 12 may be replaced by some other quantity, which has the same concentration and temperature dependence. A convenient form of reduced time is

$$
t_{\mathrm{r}}=t\left(\frac{\eta_{0}^{0}}{\eta^{0}}\right)\left(\frac{c}{c_{0}}\right)^{n}
$$

where $n$ is a constant (usually $1 \leq n \leq 3$ for homopolymers; see Discussion Section) to be determined by trial and error or otherwise. By appropriate choices for values of $n$, the method of reduced variables has been successfully applied to solutions of homopolymers over various (although limited in each case) ranges of temperature and concentration.

Measurements were performed with a coneand-plate rheometer (Multipurpose Rheometer, Iwamoto Seisakusho Co., Ltd.). Description of the apparatus has been previously given. ${ }^{22}$

\section{RESULTS}

Properties of SBS "Solutions" in Liquid Paraffin When the mixture of the copolymer Solprene 411, liquid paraffin, and dichloromethane was left in the air without any stirring, a transparent and colorless gel was obtained on evaporation of the dichloromethane. If the mixture was stirred during evaporation, it became non-homogeneous on evaporation. The gel containing more than $10 \%$ of polymer was strong and restored its original shape at once on release of a large deformation, e.g., 100-\% elongation. The 20-\% "solution" had a shear elastic modulus of approximately $2 \times 10^{4} \mathrm{~Pa}$ and did not exhibit any creep behavior at room temperature. ${ }^{23}$ The gel became unstable and weak as the concentration decreased. The gel containing less than ap- 
proximately $10 \%$ of polymer showed a marked phenomenon of syneresis. The gel of concentration below $5 \%$ readily collapsed into a gruellike dispersion even with a light shaking.

\section{Zero-Shear Viscosity}

Figure 1 shows the temperature dependence of the zero-shear viscosity for solutions of the SBS copolymer in 1-chlorohexadecane (CC), di(2-ethylhexyl) phthalate (DOP), and chlorinated biphenyl (CB). All the weight percentage concentrations, $20 \%, 19 \%$, and $13.5 \%$ indicated, correspond to the same value, $1.8 \times 10^{2} \mathrm{~kg} \mathrm{~m}^{-3}$, at $20^{\circ} \mathrm{C}$ by assuming that the volumes of polymer and solvent are additive; densities of SBS, CC, DOP, and CB were $9.35 \times 10^{2}, 8.64 \times 10^{2}, 1.00 \times$ $10^{3}$, and $1.46 \times 10^{3} \mathrm{~kg} \mathrm{~m}^{-3}$, respectively, at $20^{\circ} \mathrm{C}$. The viscosities of solvents are shown in Figure 2.

For the DOP solution, $\log \eta^{0}$ varied linearly

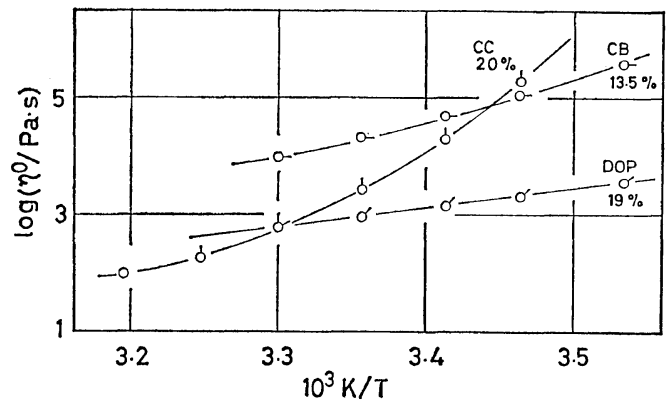

Figure 1. Temperature dependence of zero-shear viscosity for solutions of Solprene 411 in three solvents. Indicated concentrations in weight per cent approximately correspond to $1.8 \times 10^{2} \mathrm{~kg} \mathrm{~m}^{-3}$.

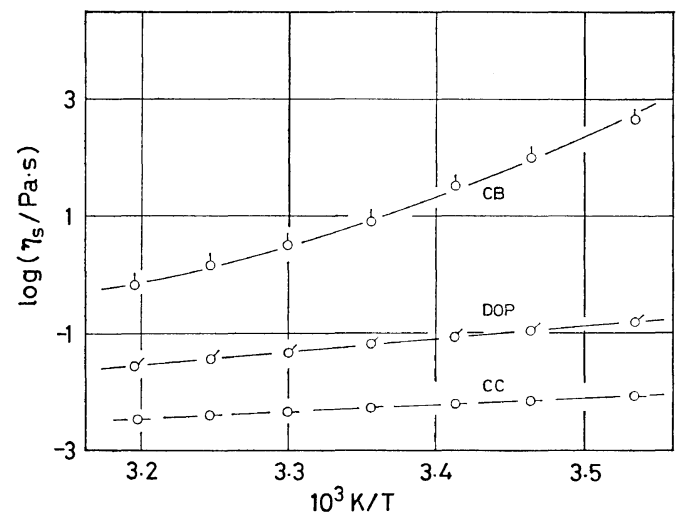

Figure 2. Temperature-dependence of viscosity of three solvents. with $1 / T$. The activation energy of flow $E$ defined by

$$
E=-R \frac{\mathrm{d} \ln \eta^{0}}{\mathrm{~d}(1 / T)}
$$

was evaluated as $66 \mathrm{~kJ} \mathrm{~mol}^{-1}$ for the DOP solution, where $R$ is the gas constant. For the CB solution, $\log \eta^{0}$ did not vary exactly linearly with $1 / T$. The activation energy if calculated by fitting a straight line to five points in Figure 1 was $112 \mathrm{~kJ} \mathrm{~mol}^{-1}$. The error may not be too large in the temperature range of measurement. On the other hand, $\log \eta^{0}$ increased much faster than it did linearly as $1 / T$ increased in the case of $\mathrm{CC}$ solution. The activation energy evaluated from the two points at high temperatures (35 and $40^{\circ} \mathrm{C}$ ) was $96 \mathrm{~kJ} \mathrm{~mol}^{-1}$ while that from lower temperatures $\left(15.8\right.$ and $\left.20^{\circ} \mathrm{C}\right)$ was $384 \mathrm{~kJ} \mathrm{~mol}^{-1}$.

Comparatively high viscosity and high activation energy of the CB solution seem to be due to the high viscosity and activation energy of the solvent. On the other hand, the high viscosity and activation energy of the CC solution cannot be attributed to the flow property of the solvent, which exhibited the lowest viscosity and activation energy among the three solvents as shown in Figure 2. Figure 3 shows the temperature dependence of the relative viscosity $\eta^{0} / \eta_{\mathrm{s}}$ calculated from the data of Figures 1 and 2. The difference between $\eta^{0} / \eta_{\mathrm{s}}$ for any pair of solvent systems was the larger at lower temperature and the value of $\eta^{0} / \eta_{\mathrm{s}}$ increased in the order $\mathrm{CB}<\mathrm{DOP}<\mathrm{CC}$ at any temperature. The order is in agreement with the order of decreasing solvent power for polystyrene. Thus, the relative viscosity increased as the amount of

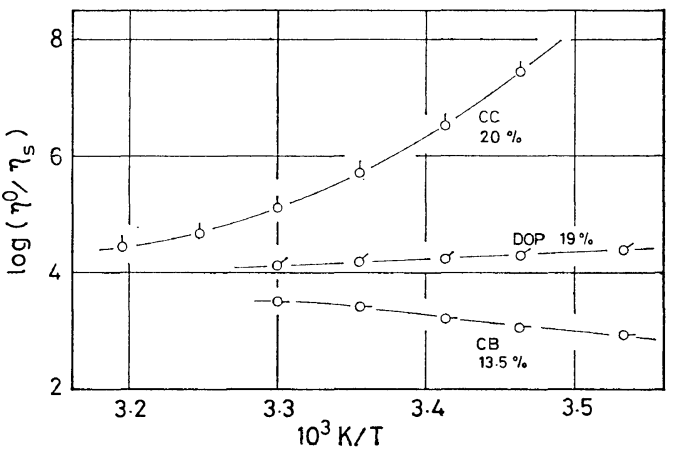

Figure 3. Relative viscosity for solutions of Solprene 411 as calculated from Figures 1 and 2 . 


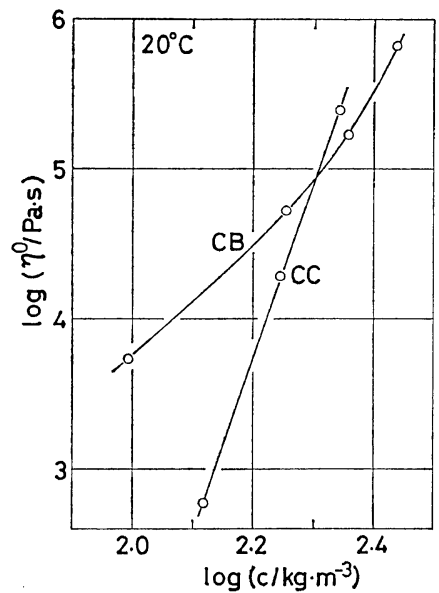

Figure 4. Concentration dependence of viscosity for solutions of Solprene 411 in two solvents at $20^{\circ} \mathrm{C}$.

solvent absorbed in the polystyrene domain decreased and the polystyrene domain became harder.

The relative viscosity $\eta^{0} / \eta_{\mathrm{s}}$ for the CB solution increased as the temperature increased in contrast with those for CC and DOP solutions. The same type of increase has been observed for solutions of rubbery materials in chlorinated biphenyl. $^{24}$ The result of Figure 3 may imply that in the CB solution the polystyrene domain, if at all, does not contribute much to the viscosity of the solution in contrast with the CC solution.

Figure 4 shows the concentration dependence of the viscosity $\eta^{0}$ for $\mathrm{CC}$ and $\mathrm{CB}$ solutions. The concentration dependence in the range of $c$ investigated was approximately described by

$$
\begin{aligned}
& \eta^{0} \propto c^{5} \text { in CB } \\
& \eta^{0} \propto c^{12} \text { in CC }
\end{aligned}
$$

The power 5 for the CB solution is of the same order as observed for various homopolymer solutions in approximately the same range of concentration. ${ }^{25}$ The power 12 for the CC solution is rather high. In the case of homopolymers, such a strong concentration dependence has been observed when either the concentration is very high or the solvent exhibits a very high viscosity and activation energy of flow.

\section{Linear Viscoelasticity of $C B$ Solutions}

The linear viscosity growth function $\bar{\eta}(t)$ and the viscosity decay function $\tilde{\eta}(t)$ are shown in Figures 5 and 6 , respectively, for the copolymer Solprene 411 solutions in chlorinated biphenyl (CB) of various concentrations. The data in these figures were obtained at various temperatures and reduced to the reference temperature $30^{\circ} \mathrm{C}$ by the method of reduced variables, eq 11 and 13. Since the reduction was performed here with regard to time and temperature, $c_{0}$ was set equal to $c$ so that $t_{\mathrm{r}}=t \eta_{0}^{0} / \eta^{0}$. Apparently, the data at various temperatures support a com-

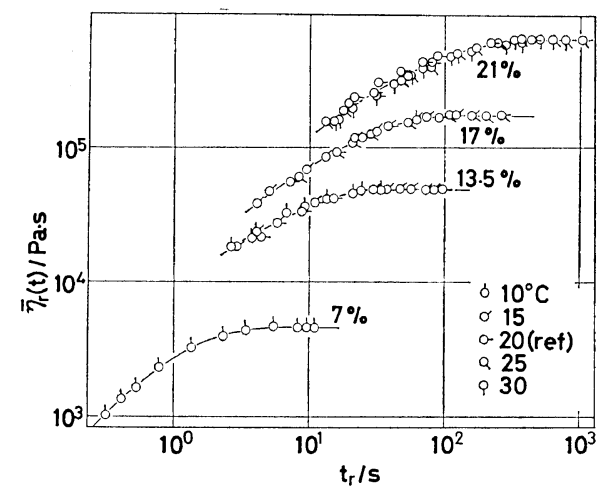

Figure 5. Linear viscosity growth function reduced to $20^{\circ} \mathrm{C}$ for solutions of Solprene 411 in chlorinated biphenyl. Various directions of pips represent temperatures of measurement. Concentrations indicated in weight per cent correspond to $2.74 \times 10^{2}$, $2.27 \times 10^{2}, 1.84 \times 10^{2}$, and $0.98 \times 10^{2} \mathrm{~kg} \mathrm{~m}^{-3}$ from top to bottom.

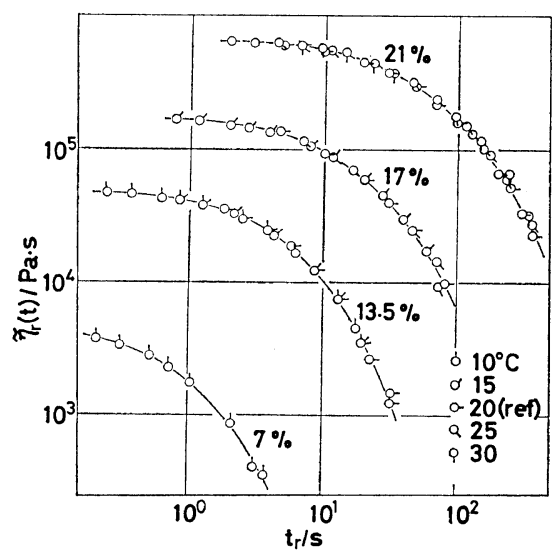

Figure 6. Linear viscosity decay function reduced to $20^{\circ} \mathrm{C}$ for solutions of Solprene 411 in chlorinated biphenyl. Directions of pips and concentrations are the same as indicated in caption for Figure 5. 
mon curve at each concentration. The data for the $7-\%$ solution, obtained at $10^{\circ} \mathrm{C}$ and reduced to $30^{\circ} \mathrm{C}$, were also included in Figures 5 and 6 . Thus, the SBS solutions in CB were thermorheologically simple in the measured ranges of concentration and temperature. Incidentally, the phenomenological relation of eq 5 was supported by the data of Figures 5 and 6 as well as by all the data shown subsequently.

In order to examine the effect of varying concentration on the functions $\bar{\eta}(t)$ and $\tilde{\eta}(t)$, we applied the method of reduced variables with respect to time and concentration. We applied eq 11 and 13 to the data of Figures 5 and 6 and chose $c_{0}=2.92 \times 10^{2} \mathrm{~kg} \mathrm{~m}^{-3}$ and $n=1$. The results are shown in Figure 7. Here neither of the reduced functions support a common curve. Any other choice of an $n$ value (which means horizontal shift of curves) did not lead to superposition over the whole time range investigated. Thus, the time-concentration reduction rule was not applicable to the SBS solutions in CB.

In Figure 7 the deviation of the reduced functions $\bar{\eta}_{r}$ is noticeable at intermediate times and that of $\tilde{\eta}_{\mathrm{r}}$ at relatively long times. (The convergence of curves at long times for $\bar{\eta}_{\mathrm{r}}$ or at short times for $\tilde{\eta}_{\mathrm{r}}$ is due to the definition of reduced functions and is not of any special importance.) These deviations are due to the

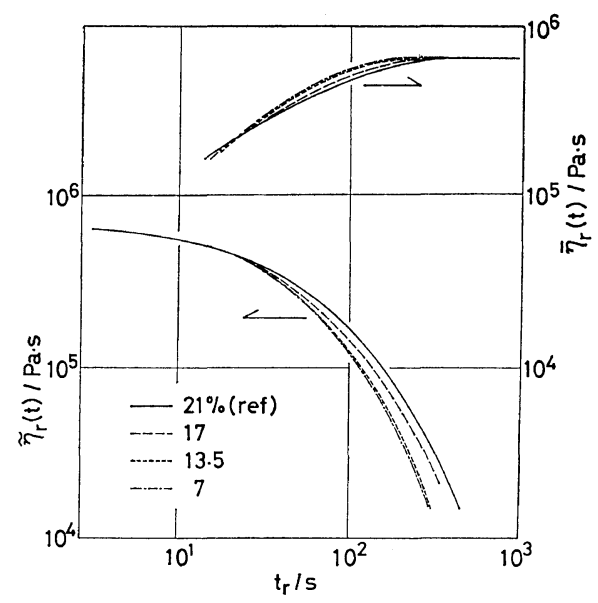

Figure 7. Viscosity growth function $\bar{\eta}_{\mathrm{r}}$ and viscosity decay function $\tilde{\eta}_{\mathrm{r}}$ reduced to reference concentration $21 \%$ for solutions of Solprene 411 in chlorinated biphenyl at $20^{\circ} \mathrm{C}$. Curves at various concentrations are taken from Figures 5 and 6. variation in the shape of curves with varying concentration. The viscosity growth function $\bar{\eta}_{\mathrm{r}}$ of higher concentration increases more gradually over a wider range of reduced time than that of lower $c$. The viscosity decay function $\tilde{\eta}_{r}$ of higher $c$ decreases more slowly over a wider range of $t_{\mathrm{r}}$ than that of lower $c$. These results indicate that the relaxation spectrum becomes broader with a long tail at long times as the concentration increases. Concentration dependence of this type has not been reported for homopolymer solutions. In a case where the concentration-time reduction rule was not applicable in homopolymer solutions, a tail of relaxation spectrum at long times appeared as the concentration decreased. ${ }^{26}$

The location of reduced curves for $\tilde{\eta}_{\mathrm{r}}$ at long times may be regarded to represent the concentration-dependence of the maximum relaxation time $\tau_{1}{ }^{0}$. By choosing a suitable value for $n$, one can make the reduced curves to superimpose on one another at the long time end. The concentration dependence of $\tau_{1}^{0}$ obtained with this method was

$$
\frac{\tau_{1}{ }^{0}}{\eta^{0}} \propto \frac{1}{c^{0.7}}
$$

The value of $n$ smaller than unity has been reported for polystyrene solutions of moderate concentrations, where the polymer molecules are supposed to overlap with each other but still entanglement couplings are not developed very much. $^{27}$

\section{Linear Viscoelasticity of CC Solutions}

For solutions of the copolymer Solprene 411 in 1-chlorohexadecane (CC), the measurements of the viscoelastic functions $\bar{\eta}(t)$ and $\tilde{\eta}(t)$ were performed over a very limited range of concentration and temperature. The reason was that the relaxation time of the solutions was very much affected by $c$ or $T$ and became too large or too small to match the operating condition of the rheometer unless $c$ and $T$ were kept in a certain limited range. Figures 8 and 9 show, respectively, those few results of functions $\bar{\eta}(t)$ and $\tilde{\eta}(t)$, which were obtained by direct measurements.

Figure 10 gives the reduced plots of $\bar{\eta}(t)$ and $\tilde{\eta}(t)$ for the $25-\%\left(2.20 \mathrm{~kg} \mathrm{~m}^{-3}\right.$ at $\left.20^{\circ} \mathrm{C}\right)$ solution to examine applicability of the time-temperature 
reduction rule. The reference temperature was taken as $25^{\circ} \mathrm{C}$ and $c_{0}$ was put equal to $c$ in eq 11 and 13. The reduced curves at various temperatures did not superimpose on one another to form single master curves for $\bar{\eta}_{\mathrm{r}}(t)$ or $\tilde{\eta}_{\mathrm{r}}(t)$. Thus, the SBS solution in CC was not thermorheologically simple.

It may be noted that the reduced curves for $\tilde{\eta}(t)$ as well as for $\bar{\eta}(t)$ had a tendency to superimpose with one another in the range of long times. This result indicates that the maximum

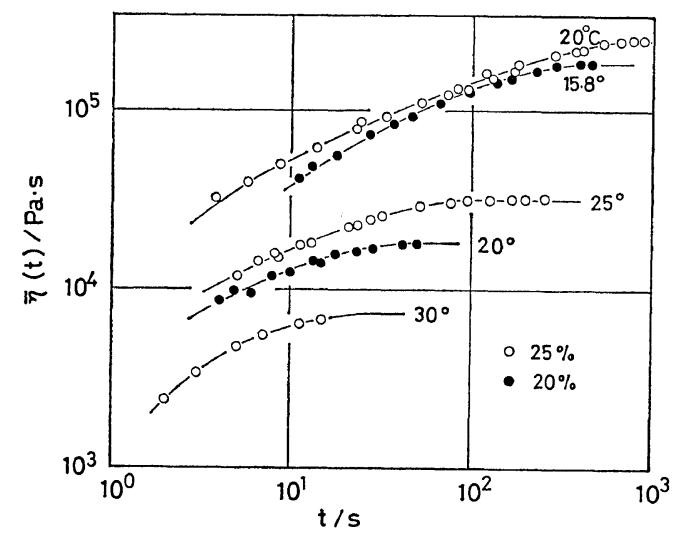

Figure 8. Linear viscosity growth functions for 20 and $25-\%\left(1.75 \times 10^{2}\right.$ and $2.20 \times 10^{2} \mathrm{~kg} \mathrm{~m}^{-3}$, respectively, at $20^{\circ} \mathrm{C}$ ) solutions of Solprene 411 in 1-chlorohexadecane at various temperatures.

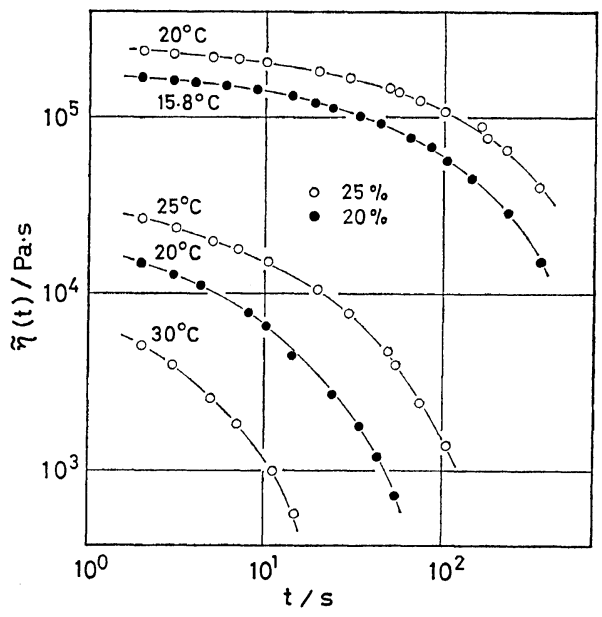

Figure 9. Linear viscosity decay functions for 20 and $25-\%\left(1.75 \times 10^{2}\right.$ and $2.20 \times 10^{2} \mathrm{~kg} \mathrm{~m}^{-3}$, respectively, at $20^{\circ} \mathrm{C}$ ) solutions of Solprene 411 in 1chlorohexadecane at various temperatures.

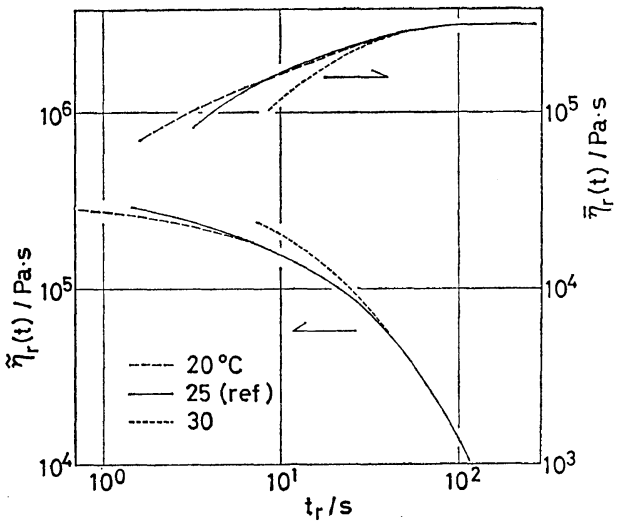

Figure 10. Linear viscosity growth function $\bar{\eta}_{r}$ and viscosity decay function $\tilde{\eta}_{r}$ reduced to $25^{\circ} \mathrm{C}$ for $25-\%$ solution of Solprene 411 in 1-chlorohexadecane. Curves are taken from Figures 8 and 9.

relaxation time is proportional to the zero-shear viscosity, i.e.,

$$
\frac{\tau_{1}{ }^{0}}{\eta^{0}}=\text { const } \quad \text { (independent of } T \text { ) }
$$

It may also be noted that the reduced curves for various temperatures were different in shape from one another. At high temperatures the viscosity growth function $\bar{\eta}_{\mathrm{r}}(t)$ rapidly approached the steady value in a limited range of time and the viscosity decay function $\tilde{\eta}_{\mathbf{r}}(t)$ fell rapidly over a limited range of long reduced times. On the other hand, the stress grew or lessened gradually over a wide range of reduced time at low temperatures. These features indicate that the relaxation spectrum at a high temperature exhibits an abrupt fall at the long time end while that at a low temperature gradually decreases with relaxation time until it becomes very low at the long time end (see Figure 11). The data for the 20-\% solution led to a figure similar to Figure 10 and to the same conclusion stated above concerning the maximum relaxation time and the shape of relaxation spectrum.

Figure 12 shows the reduced plots with respect to time and concentration for the SBS solutions in $\mathrm{CC}$ at $20^{\circ} \mathrm{C}$. The reduced functions $\bar{\eta}_{\mathrm{r}}(t)$ and $\tilde{\eta}_{\mathrm{r}}(t)$ calculated by eq 11 were plotted against the reduced time of eq 13 with $n=1$ (dashed curves) and $n=-1$ (dotted curves). In each case, no single master curve was obtained either 


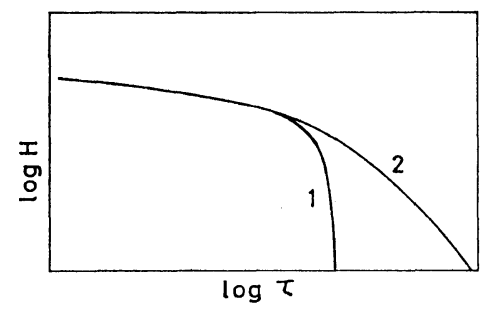

Figure 11. Logarithm of relaxation spectra $H$ schematically plotted against the logarithm of relaxation time $\tau$ with arbitrary origins for ordinate and abscissa. Curve 1 represents the behavior of solutions of SBS in chlorincted biphenyl and curve 2 in 1-chlorohexadecane. For CC solutions shape of relaxation spectrum has a tendency to vary from type 2 to 1 as concentration decreases or temperature increases.

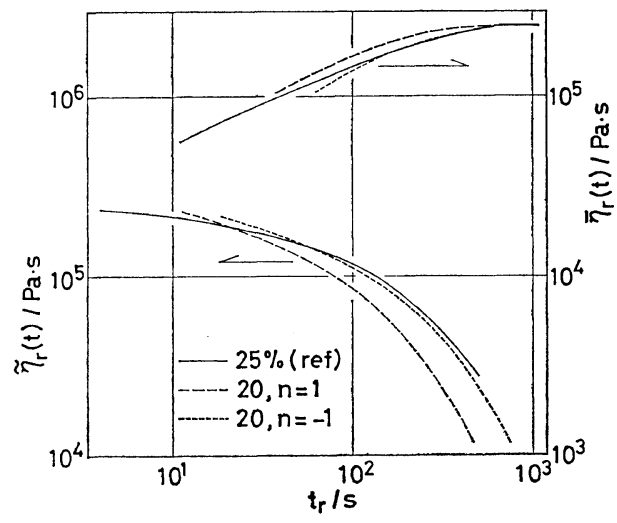

Figure 12. Linear viscosity growth function $\bar{\eta}_{r}$ and viscosity decay function $\tilde{\eta}_{\mathrm{r}}$ reduced to reference concentration $25 \%$ for solutions of Solprene 411 in 1-chlorohexadecane at $20^{\circ} \mathrm{C}$. Curves are taken from Figures 8 and 9. See text for $n$ values.

for $\bar{\eta}_{\mathrm{r}}(t)$ or $\tilde{\eta}_{\mathrm{r}}(t)$. It is seen that in a limited range of long times the reduced curves for $n=-1$ approximately superimpose on each other. This result implies that the concentration dependence of the maximum relaxation time is expressed approximately by

$$
\frac{\tau_{1}{ }^{0}}{\eta^{0}} \propto c
$$

The ratio of the left hand side has never been reported to increase with $c$ for homopolymer solutions to the knowledge of the author. The variation in shape of curves with varying con- centration is analogous to that with varying temperature; the increase in concentration corresponds to the decrease in temperature. This qualitative feature is shown in Figure 11. The data at $30^{\circ} \mathrm{C}$ gave the same result as deduced from Figure 12.

\section{Comparison of Solvents}

Figure 13 gives the comparison of linear viscoelastic functions for two solvents, chlorinated biphenyl (CB) and 1-chlorohexadecane (CC), obtained for the same concentration $2.36 \times 10^{2}$ $\mathrm{kg} \mathrm{m}^{-3}$ and at the same temperature $20^{\circ} \mathrm{C}$. For the CB solution, $\bar{\eta}_{\mathrm{r}}(t)=\bar{\eta}(t)$ was plotted against $t_{\mathrm{r}}=t$ and for the CC solution, $\bar{\eta}_{\mathrm{r}}(t)=\bar{\eta}(t) \eta^{0}$ (CB solution $) / \eta^{0}$ (CC solution) was plotted against $t_{\mathrm{r}}=$ $t \eta^{0}$ (CB solution) $/ \eta^{0}$ (CC solution). With the reduced plot of this type, the data for different solvents are the same in the case of homopolymer solutions. ${ }^{28}$ In Figure 13 , it can be seen that the reduced curves for two solvents do not coincide, that the ratio $\tau_{1}{ }^{0} / \eta^{0}$ for the CC solution is larger than that for the CB solution, and that the shape of curves depends on the solvent. The difference in the shape indicates the difference of the relaxation spectra as shown in Figure 11, where the CC solution corresponds to the curve 1 and the $C B$ solution to 2 .

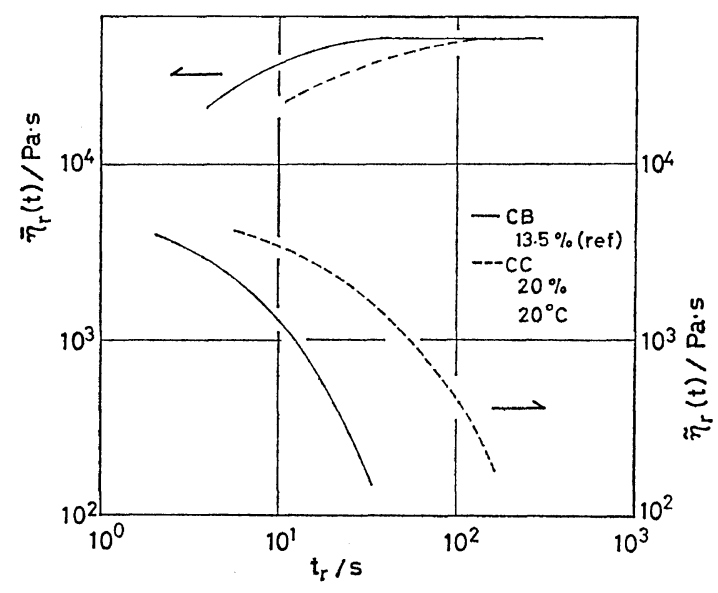

Figure 13. Linear viscosity growth function $\bar{\eta}_{\mathbf{r}}$ and viscosity decay function $\tilde{\eta}_{\mathrm{r}}$ for $1.8 \times 10^{2} \mathrm{~kg} \mathrm{~m}^{-3}$ solutions of Solprene 411 in two solvents at $20^{\circ} \mathrm{C}$. Curves for $\mathrm{CC}$ solutions are reduced to hypothetical reference state in which $C C$ solvent had the same viscosity as $\mathrm{CB}$ solvent. 
Rheology of Copolymer Solutions. III.

\section{DISCUSSION}

\section{Summary of Results}

For most of the homopolymer solutions the shape of relaxation spectrum in the plot of $\log H$ vs. $\log \tau$ is not much affected by the concentration or temperature. Therefore the method of reduced variables with respect to $t$ and $T$ or $t$ and $c$ is applicable unless the data for a very wide range of $c$ are concerned. The viscosity and the maximum relaxation time for homopolymer solutions may be described in a rather neat scheme irrespective of the species of polymer or solvent: ${ }^{29}$

$$
\begin{array}{ll}
\eta^{0} \propto \zeta c M, & \frac{\tau_{1}^{0}}{\eta^{0}} \propto \frac{M}{c} \\
\eta^{0} \propto \zeta(c M)^{3.5}, & \frac{\tau_{1}^{0}}{\eta^{0}} \propto \frac{M}{c} \\
\eta^{0} \propto \zeta(c M)^{3.5}, & \frac{\tau_{1}^{0}}{\eta^{0}} \propto \frac{1}{c^{n}}
\end{array}
$$

where $M$ is the molecular weight, $\zeta$ is the friction coefficient of segment, and $n$ is a constant. Equation 20 is applicable to solutions of relatively low molecular weight or low concentration, at which entanglement coupling does not exist. Equation 22 is applied to solutions of high molecular weight and concentration, at which the temporary network system due to the entanglement coupling is well developed and plays the main role in originating stress in flow. The reported values of $n$ lie between 2 and 3. It is believed that there is a range of $M$ and $c$ where eq 21 is applicable, in between the two ranges stated above. ${ }^{29,30}$ Throughout the eq 20-22, the friction coefficient is a function of $T$ and $c$. The friction coefficient varies in proportion to a low power of $c$, say $c^{1}-c^{2}$, unless $c$ is very high or the solvent viscosity is very high. Thus the viscosity $\eta^{0}$ is ordinarily proportional to $c^{5}$ for many polymer solutions of $10-20-\%$ concentration. The theory of De-Gennes ${ }^{31}$ predicts that the friction coefficient is proportional to $c^{1.5}$.

The linear viscoelastic behavior of the SBS solution in chlorinated biphenyl exhibited little but recognizable difference from that of the homopolymer solutions as far as the effect of temperature and concentration was concerned; the time-concentration reduction rule was not applicable and the concentration dependence of the maximum relaxation time (eq 17) was slightly different from that of eq 20 or 21 . The shape of the relaxation spectrum varied with concentration in a manner similar to the CC solution but to a lesser extent.

On the other hand, in the case of SBS solutions in $\mathrm{CC}$ the effect of varying temperature and concentration on linear viscoelastic behavior was entirely different from that of homopolymer solutions. The method of reduced variables with respect to $t$ and $T$ as well as to $t$ and $c$ was not applicable. The zero-shear viscosity exhibited a very strong temperature dependence and a strong concentration dependence. The ratio $\tau_{1}^{0} / \eta^{0}$ was larger than that for the CB solution of the same concentration and increased with $c$ in contrast with all the expected types, eq 20-22, of concentration dependence for homopolymer solutions. The shape of the relaxation spectrum varied with $c, T$, and the solvent as shown in Figure 11. The shape of the relaxation time represented by the curve 2 of Figure 11 is usually assigned to polymers of broad molecular weight distribution in the case of homopolymer solutions. Thus the higher $c$, lower $T$, and poorer solvent power for polystyrene may be related to some inhomogeneity or distribution in size of flow unit in SBS solutions.

A part of the present result may be compared with that of Kotaka and White. ${ }^{8}$ Since the quantity $\lambda \equiv J_{\mathrm{e}}{ }^{0} \eta^{0}$ of Kotaka and White is a certain average $\left(=\sum_{p} G_{\mathrm{p}}{ }^{0}\left(\tau_{\mathrm{p}}{ }^{0}\right)^{2} / \sum_{p} G_{\mathrm{p}}{ }^{0} \tau_{\mathrm{p}}{ }^{0}\right)$ of relaxation times, $J_{\mathrm{e}}{ }^{0}$ may be compared with $\tau_{1}{ }^{0} / \eta_{0}$. They revealed that $J_{\mathrm{e}}{ }^{0}$ increased with increasing $n$-decane, a non-solvent for polystyrene, in solutions of a styrene-butadiene di-block copolymer and an SBR in mixtures of $n$-decane and decalin. On the other hand, $J_{\theta}{ }^{0}$ was not affected by the composition of the solvent in the case of a styrene-butadiene-styrene triblock copolymer.

Paul, et al., ${ }^{5}$ examined the effect of solvent and concentration on a relaxation time $\tau^{\prime}$ evaluated from the steady shear viscosity ${ }^{29}$ for solutions of a styrene-butadiene-styrene triblock copolymer. They observed that the ratio $\tau^{\prime} / \eta^{0} c$ increased slightly with $c$ and that the viscosity $\eta^{0}$ and the ratio $\tau^{\prime} / \eta^{0}$ at a fixed concentration 
were the smaller when the solvent was a better solvent for polystyrene. These results are in qualitative agreement with ours in spite of the difference in the method of evaluation of the relaxation time.

\section{Speculation on Structure and Flow Behavior}

It is likely that in the solution of the SBS Solprene 411 , the solution of polystyrene blocks forms dispersed domains in the continuous phase of the solution of polybutadiene blocks. Hereafter we shortly say PS or PB domains in the place of domains formed by solutions of polystyrene or polybutadiene blocks. The polystyrene blocks are not likely to form large structures, at least in liquid paraffin or cetyl chloride, such as rod- or sheet-shaped domains because the volume fraction of polystyrene blocks is much smaller than that of the polybutadiene blocks. Since the molecule of Solprene 411 terminates with a polystyrene block at every chain end, each chain has to be connected to a disperse phase of PS domain at one end and to the branching point at the other. These features amount to a network structure in which polybutadiene chains are cross-linked by PS domains and branching points. The rheological behavior of the solution may be largely determined by the hardness of the PS and PB domains, especially by the former.

The observed elastomeric behavior of the SBS "solution" in liquid paraffin is consistent with the conjecture that the PS domain is very hard and practically solid in LP, in which polystyrene hardly dissolves. The amount of solvent contained in the PS domains increases in the order of $\mathrm{LP}<\mathrm{CC}<\mathrm{DOP}<\mathrm{CB}$ and so the PS domains get softer in this order. As was seen above, the viscoelastic behavior of the solutions becomes more and more analogous to that of homopolymer solutions in this order.

In the case of $\mathrm{CC}$ solutions, the amount of solvent contained in the PS domains may probably vary to a large extent with varying temperature (and possibly with concentration) because the polystyrene of the size of polystyrene block precipitates from the solution below some temperature near the range of temperature studied. Thus the PS domains should be quite hard at low temperatures in the case of $\mathrm{CC}$ solutions.
The activation energy of flow evaluated from the viscosity in the temperature range of 15.8 to $20^{\circ} \mathrm{C}$ was $384 \mathrm{~kJ} \mathrm{~mol}^{-1}$. According to Ferry, the activation energy of flow at the glass transition temperature $T_{\mathrm{g}}$ is proportional to $T_{\mathrm{g}}{ }^{2}$ irrespective of the polymer species and is 405 $\mathrm{kJ} \mathrm{mol}^{-1}$ at $250 \mathrm{~K}$ and $586 \mathrm{~kJ} \mathrm{~mol}^{-1}$ at $300 \mathrm{~K}$. Thus, the value obtained for the SBS solution in CC is not much smaller than that which ordinary polymers exhibit at the glass transition temperature. Since diluted polybutadiene must have a very low glass transition temperature, the result implies that the stress in flow of the solution is mostly supported by the hard domains of highly concentrated polystyrene block.

The ratio $\tau_{1}^{0} / \eta^{0}$ is believed to be inversely proportional to the number of flow units, i.e., polymer molecules in unentangled systems or entanglement points, in unit volume. The result of eq 19 for the SBS solution in CC may indicate that the number of flow unit decreases with increasing concentration. The result might be interpreted in terms of the assumption of "molecular aggregated." Suppose the hard PS domains connect a few molecules to form an aggregate, whose "molecular weight" is proportional to $c^{2}$. Then eq 20 or 21 leads to

$$
\frac{\tau_{1}^{0}}{\eta^{0}} \propto c
$$

in agreement with eq 19 . In addition, eq 21 gives

$$
\eta^{0} \propto \zeta c^{10.5}
$$

which agrees with the observed result of eq 16 if a concentration dependence of the friction coefficient is assumed to be $\zeta \propto c^{1.5}$, which may be a fair guess. ${ }^{31}$ The SBS solution in CC may be regarded as a solution of molecular aggregate formed by a few polymer molecules connected by the PS domains and inter-aggregate interaction of the type of entanglement coupling is not dominant. This conjecture which follows from the nature of eq 21, may not be unreasonable for the following reason. Most of the polymer chains in an aggregate may be connected to PS domains and so the number of free chains to make entanglement coupling with chains of other aggregates may be very few in spite of the large size of the supposed aggregate. The variation 
Rheology of Copolymer Solutions. III.

of the shape of relaxation spectrum as shown in Figure 11 might also be understood in terms of the size distribution of the aggregates.

In the case of the SBS solutions in chlorinated biphenyl, the effect of temperature and concentration on linear viscoelasticity is rather close to that of homopolymer solutions. This slight difference may be due to the slight variation of the hardness of the PS phase with varying concentration. When the hardnesses of the PS and PB domains, if existent, are close to each other, then the flow behavior may not be affected much by the existence of the domain structure. Thus it is hard to tell from our result whether domain structure exists or not in chlorinated biphenyl.

Acknowledgment. Bong-Shik Kim expresses his gratitude to the Japan Ministry of Education for the grant for foreign students. This study is partly supported by a grant for Scientific Research D-165212 (1976) from the Ministry of Education.

\section{REFERENCES}

1. D. J. Meier, "Block and Graft Copolymers," J. J. Burke and V. Weiss Ed., Syracuse University Press, 1973, p 105.

2. A. E. Skoulios, ibid., p 121.

3. G. Kraus and J. T. Gruver, J. Appl. Polym. Sci., 11, 2121 (1967).

4. K. R. Arnold and D. J. Meier, J. Appl. Polym. Sci., 14, 427 (1970).

5. D. R. Paul, J. E. St. Laurence, and J. H. Troell, Polym. Eng. Sci., 2, 70 (1970).

6. G. Kraus and K. W. Rollmann, Ang. Macrom. Chem., 16/17 (Nr. 199), 271 (1971).

7. G. Kraus, F.E. Naylor, and W. Rollmann, J. Polym. Sci., Part A-2, 9, 1839 (1971).

8. T. Kotaka and J. L. White, Trans. Soc. Rheol., 17, 587 (1973).

9. N. Nemoto, K. Okawa, and H. Odani, Bull. Inst. Chem. Res., Kyoto Univ., 51, 118 (1973).

10. F. N. Cogswell and D. E. Hanson, Polymer, 16, 936 (1975).
11. C. I. Chung and J.C. Gale, J. Polym. Sci., Polym. Phys. Ed., 14, 1149 (1976).

12. See for example, J. Meissner, Pure Appl. Chem., 42, 552 (1975); J. Meissner, Proceedings of the 7th International Congress on Rheology, Gothenberg, 1976, p 85; K. Osaki, ibid., p 104.

13. See for example, T. Matsumoto, C. Hitomi, and S. Onogi, Trans. Soc. Rheol., 19, 541 (1975).

14. See for example, M. Yamamoto, "Buttaino-Henkeigaku," Seibundo-Shinkosha, Tokyo, 1972, Chapter 6.

15. B. S. Kim, K. Osaki, and M. Kurata, J. Soc. Rheol. Jpn., 4, 16 (1976).

16. K. Osaki, B. S. Kim, N. Bessho, and M. Kurata, ibid., 4, 21 (1976).

17. J. Hans and T. C. Middlebrook, Rubber World, 27, January (1973).

18. K. Taira, N. Nemoto, H. Odani, and M. Kurata, to be published.

19. See for example, J. D. Ferry, "Viscoelastic Properties of Polymers," 2nd ed, John Wiley \& Sons, New York, N.Y., 1970, Chapters 3 and 4.

20. K. Osaki, A. Murai, N. Bessho, and B. S. Kim, J. Soc. Rheol. Jpn., 4, 166 (1976).

21. See for example, H. Markovitz, J. Polym. Sci. Polym. Symp., No. 50, 431 (1975); see also Chapter 11 of ref 19.

22. K. Osaki, Y. Einaga, N. Yamada, and M. Kurata, Polym. J., 6, 72 (1974).

23. T. Kimura, K. Osaki, and M. Kurata, unpublished result.

24. N. Nemoto, T. Ogawa, H. Odani, and M. Kurata, Macromolecules, 5, 641 (1972); K. Osaki, unpublished result.

25. See for example, Chapter 17 of ref 19.

26. K. Osaki, M. Fukuda, and M. Kurata, $J$. Polym. Sci., Polym. Phys. Ed., 13, 775 (1975).

27. K. Osaki, M. Kurata, and M. Tamura, Polym. J., 1, 334 (1970).

28. M. Tanaka, K. Osaki, M. Kurata, and M. Tamura, unpublished result.

29. W. W. Graessley, Adv. Polym. Sci., 16, 1 (1974).

30. H. Odani, N. Nemoto, and M. Kurata, Bull. Chem. Res., Kyoto Univ., 50, 117 (1972).

31. P. G. De Gennes, Macromolecules, 9,594 (1976).

32. Chapter 11 of ref 9 . 